\title{
Identification of Fetal Cardiac Timing Events by Swarm Decomposition of Doppler Cardiogram Signal
}

\author{
Saeed Alnuaimi ${ }^{1}$, Shihab Jimaa ${ }^{1}$, Yoshitaka Kimura ${ }^{2}$, Georgios K Apostolidis ${ }^{3}$, Leontios J \\ Hadjileontiadis $^{1}$, Ahsan H Khandoker ${ }^{1}$ \\ ${ }^{1}$ Khalifa University, Abu Dhabi, United Arab Emirates \\ ${ }^{2}$ Tohoku University, Sendai, Japan \\ ${ }^{3}$ Aristotle University of Thessaloniki, Thessaloniki, Greece
}

\begin{abstract}
Early diagnosis of the cardiac abnormalities during the pregnancy may reduce the risk of perinatal morbidity and mortality. Doppler ultrasound signals (DUS), which is commonly used for monitoring the fetal heart rate, can also be used for identifying the event timing of fetal cardiac valve motions. In this paper, we propose a non-invasive technique to identify the fetal cardiac timing events on the basis of analysis of fetal DUS (5 normal subjects, in both early and late gestational ages). We proposed using the swarm decomposition technique which enabled the frequency contents of the Doppler signals to be linked to the opening and closing of the heart's valves (aortic and mitral). In the early gestational age, the time intervals from $R$ peak of fetal ECG to opening and closing of aortic valve were found to be $59.4 \pm 1.9 \mathrm{~ms}$ and $218.8 \pm 2.4 \mathrm{~ms}$ respectively and in the late gestational age $65.4 \pm 10 \mathrm{~ms}$ and $218.1 \pm 3.4 \mathrm{~ms}$ respectively. The rest of the identified timing were mentioned in the results and discussion section. Decomposing the fetal Doppler signal using the swarm intelligence achieved an excellent extraction of the fetal cardiac timing events.
\end{abstract}

\section{Introduction}

The Fetal heart rate (FHR) monitoring has been extensively used to assess fetal well-being. Statics state that yearly one out of 125 baby is born with some kind of congenital cardiac defects [1]. The information provided from the fetal cardiac assessments can be used for early diagnosis of fetal cardiac diseases, such as various types of Congenital Heart Diseases (CHDs) [2]. These Diseases may threaten the life of the fetus if left without any early diagnosis and treatment. The early diagnosis reduces perinatal morbidity and mortality, and provides remarkable medical and economic benefits $[3,4]$. Current techniques which provide sensitive markers for fetal cardiac assessment are generally used to monitor fetal heart rate, which are useful methods, but not enough for a full assessment of fetal cardiac activity. Fetal cardiac valve motion timings can be detected non-invasively from either fetal echocardiography or Doppler Ultrasound (DUS).

Fetal echocardiography is an expensive method, and requires skilled specialists to operate it and is only performed for particular cases of fetal and maternal conditions. Furthermore, the DUS technique which is noninvasive technique, not sophisticated and requires less skills is more suitable for this assessment. In DUS, the Doppler shift of the ultrasound beam which is reflected from moving valves of the fetal heart, manifests the movement of the cardiac valves [5-7]. The main disadvantage of DUS is the high sensitivity to fetal movement as the detection of FHR mostly relies on the correct positioning of the ultrasound probe. Fetal movement, particularly in early gestational ages, often results in signals loss and consequently leads to misdiagnosis. This paper aims to address the challenges by analyzing the fetal cardiac Doppler signals to identify the valve motion. In order to identify the valve motion using the DUS signal, the components corresponding to valves motion should be extracted from the components related to the movement of the fetal cardiac wall, valves or other organs [8].

In the 1980 s, several studies proposed noninvasive techniques which mainly aimed to analyze the systolic time intervals, using both non-invasive abdominal ECG and the DUS signal $[7,9,10]$. Short Time Fourier Transform (STFT) analysis of the DUS signal showed that the components with a higher frequency band linked to cardiac valve movement, while the low frequency components were linked to the cardiac wall motion [6]. Furthermore, multiresolution wavelet analysis technique was proposed to decompose the DUS signals with variable spectral characteristic over time using the wavelet analysis signals [11]. Empirical Mode Decomposition (EMD) was also proposed as a decomposition technique which is used for decomposing nonlinear and non-stationary time series. This algorithm can effectively separate the components 
linked to valve movements [12]. This technique has been extensively used in image, speech as well as biomedical signal processing $[13,14]$.

The authors published a review paper that reviewed the recent methods for monitoring the fetal heart rate specifically identifying the event timing of fetal cardiac valve motions [15]. In this paper, the swarm decomposition method is proposed to be used to analyze the fetal Doppler signals to identify the fetal cardiac timing events.

\section{Material and methods}

\subsection{Data}

The DUS signals were collected by $1.5 \mathrm{MHz}$ Ultrasonic Transducer 5700. Doppler ultrasound signals were recorded simultaneously from 10 pregnant women with normal single pregnancy at the gestational age of 16 to 41 $(33 \pm 6)$ weeks at Tohoku University Hospital, Japan. The extracted DUS signal components were compared in two age groups of early gestation (16-30 weeks) and late gestation (30-41 weeks), including 5 fetuses in each age group. All recordings were $1 \mathrm{~min}$ length and sampled at 1 $\mathrm{kHz}$ with 16-bit resolution. The study protocol was approved by Tohoku University Institutional Review Board and written informed consent was obtained from all participants.

\subsection{The fetal cardiac timing events and its link with the Doppler ultrasound}

An example of fetal cardiac ultrasound signal is shown in Figure 1, the FECG signal was added to the figure to verify the identified fetal cardiac timing events in the DUS signal. It can be seen clearly that the content of DUS signals varies on a beat to beat basis. DUS signals were used to monitor the fetal cardiac valve movements. From extensive research studies of the heart's physiology that have already been performed the prominent cardiac motions that occur can be realized. These motions are due to the heart wall movement and valves movement.

Figure 1 shows the relative timing of the heart's valves $\left(A_{c}, A_{o}, M_{c}\right.$, and $\left.M_{o}\right)$ and wall movements (Atrial wall contraction and Ventricle wall contraction) in relation to the reference signal FECG. Doppler frequency shifts associated with the fetal cardiac activity can be visualized. Due to the variation of the transducer-to-foetus orientation, the structures returning the largest signal will vary. DUS signals are variable in time, this being due mainly to variations in the relative orientation of the foetal heart and the ultrasound transducer [5]. Apparently, the fetal movement will therefore significantly affect the detected DUS signal. Hence, all six cardiac timing events (four valve motions and two wall motions) are unlikely to be distinguished in each cardiac cycle [16].

(A)

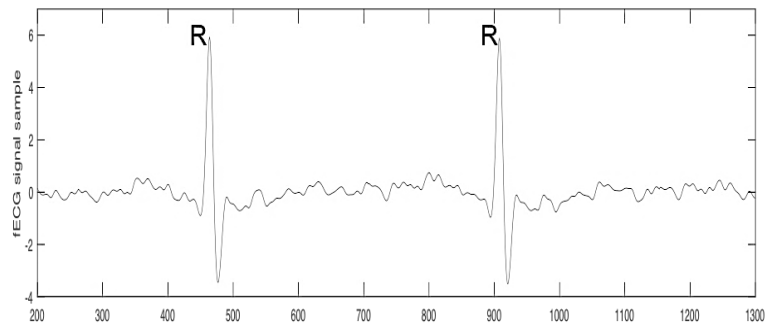

(B)

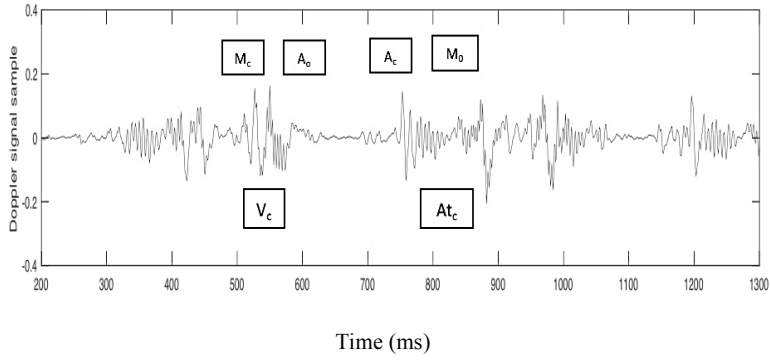

Figure 1. A sample of simultaneously recorded fetal ECG and Doppler ultrasound (DUS) signal. (A) Fetal ECG signal (B) Doppler ultrasound from fetal heart. Annotations showing the cardiac timing events of the opening and closing of the fetal heart's valves with reference to the ECG signal. Mitral opening $\left(\mathrm{M}_{\mathrm{o}}\right)$, Aortic opening $\left(\mathrm{A}_{\mathrm{o}}\right)$, Mitral closing $\left(\mathrm{M}_{\mathrm{c}}\right)$, Aortic closing $\left(\mathrm{A}_{\mathrm{c}}\right), \mathrm{At}_{\mathrm{c}}$, Atrial wall contraction, and $\mathrm{V}_{\mathrm{c}}=$ Ventricle wall contraction.

\section{Results and discussion}

\subsection{Swarm decomposition of the fetal Doppler signals}

The fundamental objective of the non-stationary signal analysis is to decompose the signal over a set of functions, these functions are localized both in time and frequency. In this decomposition technique, the concept of swarm filtering $(\mathrm{SwF})$ is used which is a discrete time signal processing algorithm that is based on a swarming model. This decomposition method is based on properly parameterizing swarm filtering, that generates oscillatory components (OCs) from a multi-component input signal. It consists of an iterative execution of a sifting-like process, the dominant oscillatory component is initially guessed then this oscillatory component is obtained by consecutive applications of swarm filtering. After that, the oscillatory component is aligned with and it is subtracted from the rest of the multi-component input signal. Lastly, when the remaining input signal does not contain any oscillatory mode of sufficient energy, the swarm decomposition process is terminated [17].

In this paper, the total number of 10 different datasets of DUS was used for testing the algorithm and obtaining the 
cardiac timings. The cardiac valves timings are Aortic opening (Ao), Aortic closing (Ac), Mitral opening (Mo) and Mitral closing $(\mathrm{Mc})$. The fetal cardiac Doppler signal was decomposed by using the swarm decomposition method and after the decomposition process the extracted oscillatory components (OCs) from the fetal cardiac Doppler signal are illustrated in both time and frequency domains as shown in Figure 2. Decomposing the fetal Doppler signal using the swarm intelligence achieved an excellent extraction of the fetal cardiac timing events. The extracted IMFs are linked with opening and closing of the mitral valves.

In order to detect the peak timings of valve motion events, the time durations within each RR interval chosen for each event were $0.000 \sim 0.06 \mathrm{sec}$ for Mc, $0.06 \sim 0.18$ $\mathrm{sec}$ for Ao, $0.18 \sim 0.26 \mathrm{sec}$ for Ac and $0.26 \sim 0.33$ for Mo [16]. Table 1 shows the mean time interval of each of the cardiac events (e.g. cardiac valves opening and closing timings from R peak of Fetal ECG namely R-A $A_{c}, R-A_{o}$, R$M_{c}$, and R-M $M_{o}$ within each RR interval for both early and late gestational ages.

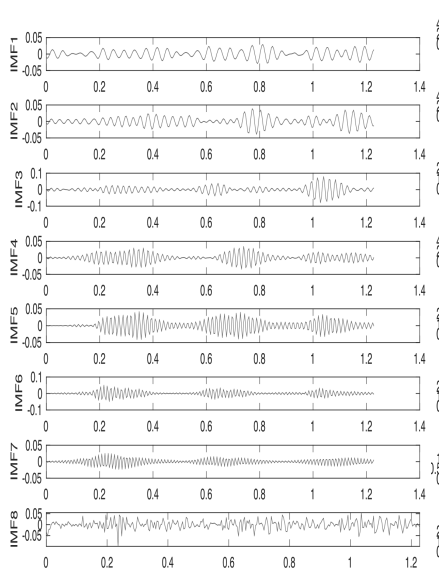

(A)

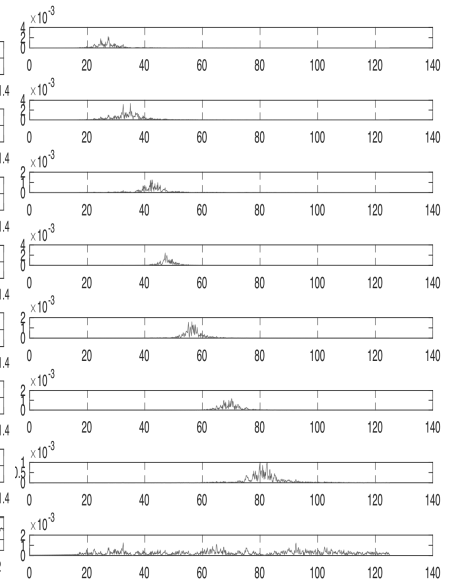

(B)
Figure 2. The extracted components from the fetal cardiac Doppler signal using SWD are shown in (A) and (B) in time and frequency domain, respectively

Table 1. Summary of time intervals of opening and closing of the heart's valves from $\mathrm{R}$ peak over each cardiac cycle.

\begin{tabular}{|c|c|c|c|c|c|c|}
\hline & & R-R & R-Mc & R-Ao & R-Ac & R-Mo \\
\hline \multirow{2}{*}{$\begin{array}{c}\text { Early } \\
\text { gestational } \\
\text { age }\end{array}$} & $\begin{array}{c}\text { Mean } \\
\text { (ms) }\end{array}$ & 392.7 & 24.4 & 59.4 & 218.8 & 303.9 \\
\cline { 2 - 7 } & $\begin{array}{c}\text { Standard } \\
\text { deviation }\end{array}$ & \pm 8.9 & \pm 1.1 & \pm 1.9 & \pm 2.4 & \pm 1 \\
\hline $\begin{array}{c}\text { Late } \\
\text { Gestational } \\
\text { age }\end{array}$ & $\begin{array}{c}\text { Mean } \\
\text { (ms) }\end{array}$ & 423.1 & 28.0 & 65.4 & 218.1 & 309.9 \\
\cline { 2 - 7 } & $\begin{array}{c}\text { Standard } \\
\text { deviation }\end{array}$ & \pm 29 & \pm 4.6 & \pm 10 & \pm 3.4 & \pm 6.2 \\
\hline
\end{tabular}
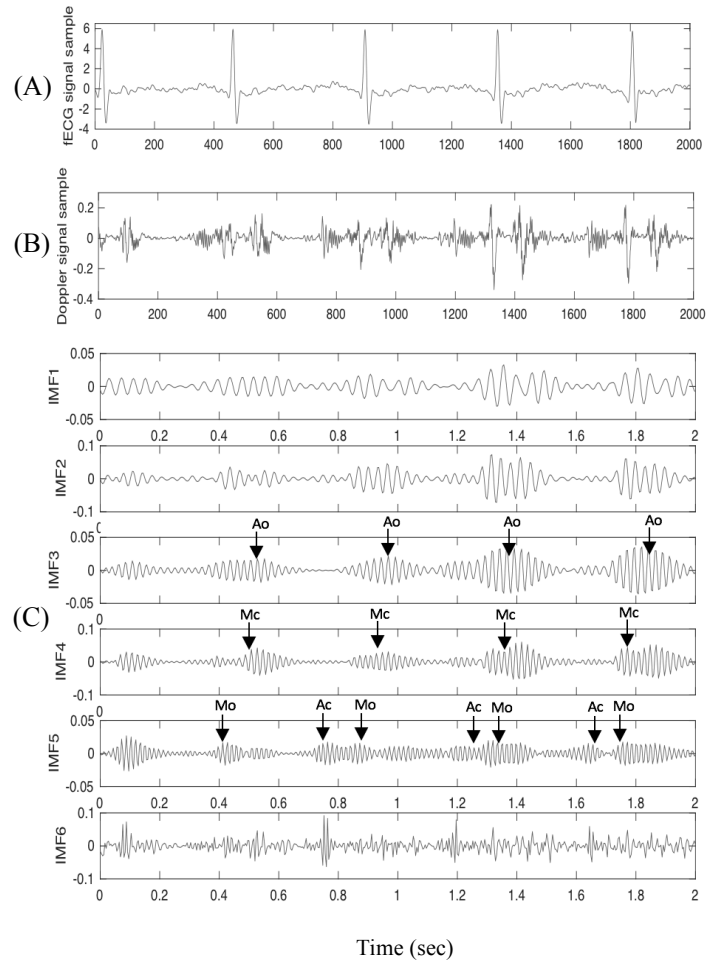

Figure 3. (A) Fetal ECG, (B) Fetal Doppler Ultrasound (DUS) signal and (C) The extracted components are obtained using swarm decomposition.

In previous studies, time intervals of the cardiac events have been estimated from the DUS signal by using several methods such as digital filtering, short term Fourier transform (STFT), or wavelet analysis $[6,18]$. The fetal DUS signal is nonlinear and non-stationary and wide changes in the signal content and spectral characteristics are noted on a beat-to-beat basis. Using fixed parameters such as cut-off frequency for filtering techniques or wavelet parameters for the whole signal and different subjects are not accurate. Thus, Swarm decomposition which is a data-driven method is more suitable for this application. Swarm decomposition has been developed for decomposing nonlinear and non- stationary signals, including the EEG signals [17] and it has not been used for this specific application before. The results show that by applying the swarm decomposition, the component which is linked to valve movements is practically separated, and its peaks which correspond to the cardiac events can be discriminated as shown in Figure 3. Figures 2 show the fetal Doppler ultrasound signal over a normal R-R interval $(0.4 \mathrm{sec})$, the extracted components using Swarm decomposition and the frequency domain of the extracted components. Table 1 shows a summary of time intervals of opening and closing of the heart's valves from $\mathrm{R}$ peak over each cardiac cycle. By comparing the estimated timings with the previous methods such as EMD in [8], it matches the time intervals of opening and closing of the heart's valves. 
The main advantage of the proposed swarm decomposition method is that it allows the efficient decomposition of a signal into a set of components that preserve physical meaning, likewise other decomposition techniques such as Empirical mode decomposition [19]. The main disadvantage of the swarm decomposition is its time complexity which although can be solved by resampling the input signal, it is still not recommended for real time applications. A limitation of this study is that the quantitative comparison with the pulsed wave Doppler image-based valve motion timings was not provided with the presented data. The quantitative comparison can be done in future studies.

\section{Conclusion}

The importance of clinical fetal heart rate monitoring has been discussed. A new decomposition method is used to analyze the fetal cardiac Doppler ultrasound signals for the fetal cardiac timing events estimation. Decomposing the fetal Doppler signal using the swarm intelligence achieved an excellent extraction of the fetal cardiac timing events. Swarm decomposition method enabled the frequency contents of the fetal Doppler signals to be linked to the opening and closing of the fetus heart's valves (aortic and mitral). The next milestone is to expand the analysis to cover large number of samples and to include the abnormal fetal cardiac Doppler signal samples.

\section{Acknowledgements}

This study was supported by grant from Buhooth program in Khalifa University, Abu Dhabi, UAE. Also the authors would like to thank the clinical support service team at Tohoku University in Japan for fetal Doppler data recordings.

\section{References}

[1] A. H. Association, "Congenital heart defects in children fact sheet," vol. 4, 2004.

[2] P. Malcus, "Antenatal fetal surveillance," Current Opinion in Obstetrics and Gynecology, vol. 16, pp. 123-128, April 2004.

[3] E. Merz, Ultrasound in Obstetrics and Gynecology: Georg Thieme Verlag, 2004.

[4] A. B. Hameed and M. S. Sklansky, "Pregnancy: maternal and fetal heart disease," Current Problems Cardiol. , vol. 32, pp. 419-494, 2007.

[5] Y. Murata and C. B. Martin, "Systolic time intervals of the fetal cardiac cycle," Obstetrics \& Gynecology, vol. 44, pp. 224-232, 1974.

[6] S. A. Shakespeare, J. A. Crowe, B. R. Hayes-Gill, K. Bhogal, and D. K. James, "The information content of doppler ultrasound signals from the fetal heart," 2001.

[7] Y. Murata, C. B. Martin, T. Ikenoue, and P. Lu,
"Antepartum evaluation of the pre-ejection period of the fetal cardiac cycle," Amer. J. Obstet. Gynecol., vol. 132, pp. 278-284, 1978

[8] F. Marzbanrad, Y. Kimura, K. Funamoto, S. Oshio, M. Endo, N. Sato, et al., "Model-based estimation of aortic and mitral valves opening and closing timings in developing human fetuses," IEEE J Biomed Health Inform, vol. 20, pp. 240-8, Jan 2016.

[9] M. B. Sampson, "Antepartum measurement of the preejection period in high-risk pregnancy," Obstetrics Gynecol, vol. 56, pp. 289-290, 1980.

[10] L. Organ, A. Bernstein, and P. Hawrylyshyn, "The preejection period as an antepartum indicator of fetal wellbeing," Amer. J. Obstetrics Gynecol., vol. 137, pp. 810 819,1980

[11] A. H. Khandoker, Y. Kimura, T. Ito, N. Sato, K. Okamura, and M. Palaniswami, "Antepartum non-invasive evaluation of opening and closing timings of the cardiac valves in fetal cardiac cycle," Med Biol Eng Comput, vol. 47, pp. 1075-82, Oct 2009.

[12] F. Marzbanrad, A. H. Khandoker, K. Funamoto, R. Sugibayashi, M. Endo, C. Velayo, et al., "Automated identification of fetal cardiac valve timings," Proc. IEEE 35th Annu. Int. Conf. Eng. Med. Biol. Soc., pp. 3893-3896, 2013.

[13] A. Bouzid and N. Ellouze, "Empirical mode decomposition of voiced speech signal," Proc. 1st IEEE Int. Symp. Control, Commun. Signal Process., pp. 603-606, 2004.

[14] J. Echeverria, J. Crowe, M. Woolfson, and B. Hayes-Gill, "Application of empirical mode decomposition to heart rate variability analysis," Med. Biol. Eng. Comput., vol. 39, pp. 471-479, 2010.

[15] S. A. Alnuaimi, S. Jimaa, and A. H. Khandoker, "Fetal cardiac doppler signal processing techniques: challenges and future research directions," Frontiers in Bioengineering and Biotechnology, vol. 5, 2017December-22 2017.

[16] A. H. Khandoker, Y. Kimura, T. Ito, and M. Palaniswami, "Non-invasive determination of electromechanical time intervals of cardiac cycle using abdominal ecg and doppler ultrasound signals from fetal hearts," Computers in Cardiology, p. 657-660, 2007.

[17] G. K. Apostolidis and L. J. Hadjileontiadis, "Swarm decomposition: A novel signal analysis using swarm intelligence," In Signal Processing, vol. 132, pp. 40-50, March 2017.

[18] T. Koga, N. Athayde, B. Trudinger, and H. Nakano, "A new and simple doppler method for measurement of fetal cardiac isovolumetric contraction time," 2001.

[19] F. Marzbanrad, Y. Kimura, K. Funamoto, R. Sugibayashi, M. Endo, T. Ito, et al., "Automated estimation of fetal cardiac timing events from Doppler ultrasound signal using hybrid models," IEEE J Biomed Health Inform, vol. 18, pp. 1169-77, Jul 2014.

Address for correspondence:

Saeed Alnuaimi

Department of Electrical and Computer Engineering, Khalifa University, Abu Dhabi, UAE

E-mail: saeed.alnuaimi@kustar.ac.ae 